\title{
SOME ASPECTS OF DYNAMIC COUPLED RESPONSE OF FUNCTIONALLY GRADED THIN-WALLED COLUMNS WITH SQUARE CROSS-SECTIONS UNDER IN-PLANE PULSE COMPRESSION
}

\author{
ZBigniew KoŁAKOWSKI \\ Lodz University of Technology, Department of Strength of Materials, Lódź, Poland; e-mail: zbigniew.kolakowski@p.lodz.pl \\ ANDRZEJ TETER \\ Lublin University of Technology, Department of Applied Mechanics, Lublin, Poland; e-mail: a.teter@pollub.pl
}

\begin{abstract}
The present paper deals with a dynamic coupled response of functionally graded columns with a quadratic cross-section subjected to an in-plane pulse loading. An Al-TiC metal-ceramic material is applied. It is assumed that functionally graded materials (FGMs) are subject to Hooke's law. The thin-walled structures are simply supported at the ends. This study is devoted to the stability problem of rectangular dynamic pulse load. The effects of temperature, wave propagation and damping are neglected. In order to obtain the equations of motion of individual plates, the classic laminate plate theory (CLPT) has been modified in such a way that it additionally accounts for all components of inertial forces. A plate model is adopted for the structures. The problem of an interaction of the global mode with the local ones is concerned (i.e., a three-modes approach). Attention has been focused on some unexpected aspects related to dynamic interactive buckling of columns having two axes of the cross-section symmetry. In the present study, a new approach to the description of this phenomenon, based on Koiter's theory, has been applied.
\end{abstract}

Keywords: FGM, dynamic response, interactive buckling, thin-walled structures, compression, pulse load

\section{Introduction}

The dynamic buckling or dynamic response takes place when a pulse load of a mean amplitude and a pulse duration comparable to the fundamental natural flexural vibration period occurs in compression of the thin-walled column. In this case, effects of damping can be neglected in practice. When the amplitude of load is high, then the structure can vibrate very strongly or can move divergently, which is caused by dynamic buckling. One can determine the critical amplitude of load using various criteria. In the literature on this problem, a lot of criteria concerning dynamic stability have been adopted. The most widely used is the Budiansky-Hutchinson criterion (Kubiak, 2007, 2013), in which it is assumed that the dynamic stability loss occurs when the maximum structure deflection grows rapidly at a small variation in the load amplitude. Other criteria have been discussed in many papers: Ari-Gur and Simonetta (1979), Petry and Fahlbusch (2000), Kubiak (2007, 2013), Teter (2011).

Dynamic global and local buckling instabilities of component functionally graded plates (the so-called FG plates) of structures subjected to conservative loads have been taken into account. The problem of an interaction of the global mode with the local ones is very interesting. The concept of interactive buckling involves the general asymptotic theory of stability. Among all versions of the general nonlinear theory, Koiter's theory (van der Heijden, 2009) of conservative systems is the most popular one (Kołakowski et al., 1999; Teter and Kołakowski, 2004; Kołakowski and Kubiak, 2005; Kołakowski and Królak, 2006). 
In the present study, the classical laminate plate theory (CLPT) (Jones, 1999; Reddy, 2004) is employed to obtain the governing equations of the thin FG plate equilibrium. In order to obtain the equations of individual plates for the asymptotic analytical-numerical method, the nonlinear theory of composite plates has been modified in such a way that it additionally accounts for forces of inertia. The differential equations of motion have been obtained from Hamilton's Principle, taking into account Lagrange's description, full Green's strain tensor, the second Piola-Kirchhoff's stress tensor and all components of inertia forces. The study is based on the numerical method of the transition matrix using Godunov's orthogonalization (Kołakowski et al., 1999; Teter and Kołakowski, 2004; Kołakowski and Kubiak, 2005; Kołakowski and Królak, 2006). A plate model of the column has been adopted in the study to describe global buckling, which leads to lowering the theoretical value of the limit load. The solution method assumed in this study enables analyzia of interactions of all buckling modes. The nonlinear equations of dynamic instability are solved with the modified Runge-Kutta method.

The nonlinear analysis of Functionally Gradient plates and shells devoted to basic types of loads is covered in the monograph by Hui-Shen (2009). The shear deformation effect is employed in the framework of Reddy's higher order shear deformation theory (HSDT) (Reddy, 2000; Reddy, 2004). Reddy (2000) presents a comparison of applications of the first order shear deformation theory (FSDT) and the classical lamination plate theory (CLPT) to functionally graded plates. The discrepancy between both theories is of $2 \%$ in the calculated deflections of the plates under analysis. The buckling and postbuckling problem of FG plates is discussed, for example, in papers by Reddy (2000), Samsam Shariata et al. (2005), Kołakowski et al. (2015). Due to the complexity of buckling problems of FG structures under compound mechanical and thermal loads, the finite element method (FEM) is the only solution possible in many cases. Therefore, in the literature, one can find many papers which present results of a solution to different problems of FG structure buckling obtained with an application of the FEM, see for example: Birman and Byrd (2007), Panda and Ray (2008), Na and Kim (2009), Kołakowski et al. (2015).

\section{Formulation of the problem}

Long thin-walled prismatic columns of length $l$, composed of plane rectangular plate segments interconnected along longitudinal edges and simply supported at both ends, are considered. All materials the FG plates are made of are subject to Hooke's law. The material properties are assumed to be temperature independent. A plate model is adopted for the structures. Wave propagation and damping effects have been neglected in the present study, as it is done in the majority of works devoted to dynamic stability. In the present study, the classical laminate plate theory (CLPT) (Jones, 1999; Reddy, 2004) is employed to obtain the governing equations of the thin FG structure equilibrium (Kołakowski and Królak, 2006; Panda and Ray, 2008). For the plate component, precise geometrical relationships are assumed in order to enable consideration of both out-of-plane and in-plane bending of the plate (Kubiak, 2007, 2013; Teter, 2007, 2011; Kołakowski et al., 1999; Teter and Kołakowski, 2001, 2004, 2005; Kołakowski and Kubiak, 2005; Kołakowski and Królak, 2006)

$$
\boldsymbol{\varepsilon}=\left\{\begin{array}{c}
\varepsilon_{x} \\
\varepsilon_{y} \\
2 \varepsilon_{x y}
\end{array}\right\}=\left\{\begin{array}{c}
u_{, x}+\frac{1}{2}\left(w_{, x}^{2}+v_{, x}^{2}+u_{, x}^{2}\right) \\
v_{, y}+\frac{1}{2}\left(w_{, y}^{2}+u_{, y}^{2}+v_{, y}^{2}\right) \\
u_{, y}+v_{, x}+w_{, x} w_{, y}+u_{, x} u_{, y}+v_{, x} v_{, y}
\end{array}\right\} \quad \boldsymbol{\kappa}=\left\{\begin{array}{c}
\kappa_{x} \\
\kappa_{y} \\
\kappa_{x y}
\end{array}\right\}=\left\{\begin{array}{c}
-w_{, x x} \\
-w_{, y y} \\
-2 w_{, x y}
\end{array}\right\}
$$

where $u, v, w$ are components of the displacement vector of the plate in the $x, y, z$ axis direction, respectively, and the plane $x y$ overlaps the mid-plane before its buckling. 
According to the rule of mixture, the properties of the functionally graded material ( $E$ - Young's modulus, $\nu$ - Poisson's ratio, $\rho$ - density) can be expressed as follows

$$
\begin{aligned}
& E(z)=E_{m}+\left(E_{c}-E_{m}\right)\left(\frac{z}{h}+\frac{1}{2}\right)^{q} \quad \nu(z)=\nu_{m}+\left(\nu_{c}-\nu_{m}\right)\left(\frac{z}{h}+\frac{1}{2}\right)^{q} \\
& \rho(z)=\rho_{m}+\left(\rho_{c}-\rho_{m}\right)\left(\frac{z}{h}+\frac{1}{2}\right)^{q}
\end{aligned}
$$

where the indices $m$ and $c$ refer to the metal and ceramic material, respectively, and $q$ is the fraction exponent.

Using the classical laminate plate theory (CLPT) (Jones, 1999; Reddy, 2004), the stress and moment resultants $(\overline{\mathbf{N}}, \overline{\mathbf{M}})$ are defined as (Jones, 1999; Teter and Kołakowski, 2005, Kołakowski and Królak, 2006)

$$
\left\{\begin{array}{l}
\overline{\mathbf{N}} \\
\overline{\mathbf{M}}
\end{array}\right\}=\left[\begin{array}{ll}
\mathbf{A} & \mathbf{B} \\
\mathbf{B} & \mathbf{D}
\end{array}\right]\left\{\begin{array}{l}
\varepsilon \\
\kappa
\end{array}\right\}
$$

where $\mathbf{A}, \mathbf{B}, \mathbf{D}$ are extensional, coupling and bending stiffness matrices, respectively, for the FG structure. Their components are listed below

$$
\begin{array}{rlrl}
A_{11} & =A_{22}=\int_{-h / 2}^{h / 2} \frac{E(z)}{1-\nu^{2}(z)} d z & A_{12}=A_{21}=\int_{-h / 2}^{h / 2} \frac{E(z) \nu(z)}{1-\nu^{2}(z)} d z \\
A_{66}=\int_{-h / 2}^{h / 2} \frac{E(z)}{2[1+\nu(z)]} d z & A_{16}=A_{61}=0 \\
B_{11}=B_{22}=\int_{-h / 2}^{h / 2} \frac{E(z)}{1-\nu^{2}(z)} z d z & B_{12}=B_{21}=\int_{-h / 2}^{h / 2} \frac{E(z) \nu(z)}{1-\nu^{2}(z)} z d z \\
B_{66}=\int_{-h / 2}^{h / 2} \frac{E(z)}{2[1+\nu(z)]} z d z & B_{16}=B_{61}=0 \\
D_{11}=D_{22}=\int_{-h / 2}^{h / 2} \frac{E(z)}{1-\nu^{2}(z)} z^{2} d z & D_{12}=D_{21}=\int_{-h / 2}^{h / 2} \frac{E(z) \nu(z)}{1-\nu^{2}(z)} z^{2} d z \\
D_{66}=\int_{-h / 2}^{h / 2} \frac{E(z)}{2[1+\nu(z)]} z^{2} d z & D_{16}=D_{61}=0
\end{array}
$$

Due to the presence of the nontrivial submatrix $\mathbf{B}$, the coupling between extensional and bending deformations exists as it is in the case of unsymmetrical laminated plates (Jones, 1999; Kołakowski et al., 2015). An extensional force results not only in extensional deformations, but also bending of the FG plate. Moreover, such a plate cannot be subjected to the moment without suffering simultaneously from extension of the middle surface.

The nonlinear problem of dynamic stability has been solved with the asymptotic perturbation method. Let $\lambda$ be a load factor. The displacement fields $\bar{U}$ and the sectional force fields $\bar{N}$ (Koiter's type expansion for the static buckling problem (van der Heijden, 2009)) have been expanded into power series with respect to the dimensionless amplitude of the $r$-th mode deflection $\zeta_{r}$ (normalized in the given case by the condition of equality of the maximum deflection to the thickness of the first component plate $h_{1}$ ) (Kołakowski et al., 1999; Teter and Kołakowski, 2001, 2004, 2005; Teter, 2007, 2011; Kołakowski and Kubiak, 2005; Kołakowski and Królak, 2006; Kubiak, 2007, 2013; van der Heijden, 2009)

$$
\begin{aligned}
& \overline{\mathbf{U}}=\lambda(t) \overline{\mathbf{U}}_{0}+\zeta_{r}(t) \overline{\mathbf{U}}_{r}+\zeta_{r}(t) \zeta_{q}(t) \overline{\mathbf{U}}_{q r}+\ldots \\
& \overline{\mathbf{N}}=\lambda(t) \overline{\mathbf{N}}_{0}+\zeta_{r}(t) \overline{\mathbf{N}}_{r}+\zeta_{r}(t) \zeta_{q}(t) \overline{\mathbf{N}}_{q r}+\ldots
\end{aligned}
$$

where the pre-buckling static fields are $\overline{\mathbf{U}}_{0}, \overline{\mathbf{N}}_{0}$, the first nonlinear order fields are $\overline{\mathbf{U}}_{r}, \overline{\mathbf{N}}_{r}$ (the eigenvalues and eigenvectors problems) and the second nonlinear order fields $-\overline{\mathbf{U}}_{q r}, \overline{\mathbf{N}}_{q r}$, 
respectively. The range of indexes is $[1, J]$, where $J$ is the number of interacting modes. The summation is supposed on the repeated indexes.

If the structure contains the geometric imperfections $\overline{\mathbf{U}}^{*}$ (only linear initial imperfections determined by the shape of $r$-th buckling modes i.e., $\left.\overline{\mathbf{U}}^{*}=\zeta_{r}^{*} \mathbf{U}_{r}\right)$, then the total energy of the structures can be written in the form (Schokker et al., 1996; Kołakowski et al., 1999; Teter and Kołakowski, 2004; Teter, 2007, 2011; Kołakowski and Kubiak, 2005; Kołakowski and Królak, 2006; Kubiak, 2007, 2013)

$$
\begin{aligned}
\Pi= & -\frac{1}{2} \sigma^{2}(t) \bar{a}_{0}+\frac{1}{2} \sum_{r=1}^{J} \bar{a}_{r} \zeta_{r}^{2}(t)\left(1-\frac{\sigma(t)}{\sigma_{r}}\right)+\frac{1}{3} \sum_{p}^{J} \sum_{q}^{J} \sum_{r}^{J} \bar{a}_{p q r} \zeta_{p}(t) \zeta_{q}(t) \zeta_{r}(t) \\
& +\frac{1}{4} \sum_{r}^{J} \bar{b}_{r r r r} \zeta_{r}^{4}(t)-\sum_{r}^{J} \frac{\sigma(t)}{\sigma_{r}} \bar{a}_{r} \zeta_{r}^{*} \zeta_{r}(t)+\frac{1}{2} \sum_{r}^{J} m_{r} \zeta_{r, t}^{2}(t)
\end{aligned}
$$

Then, Hamilton's principle leads to the following Lagrange's equation (i.e. equations of motion)

$$
\zeta_{r, t t}+\Omega_{r}^{2} \zeta_{r}+\omega_{r}^{2}\left(a_{p q r} \zeta_{p} \zeta_{q}+b_{r r r r} \zeta_{r}^{3}-\frac{\sigma}{\sigma_{r}} \zeta_{r}^{*}+\ldots\right)=0 \quad \text { for } \quad r=1, \ldots, J
$$

where $\zeta_{r}$ is the dimensionless amplitude of the $r$-th buckling mode, $\sigma_{r}, \omega_{r}, \zeta_{r}^{*}$ - critical stress instead of the load parameter $\lambda_{r}$ of the $r$-th buckling mode, circular frequency of free vibrations and dimensionless amplitude of the initial deflection corresponding to the $r$-th buckling mode, respectively. In equations of motion (2.7), the inertia forces of the pre-buckling state and the second order state have been neglected (Sridharan et al., 1984; Schokker, 1996; Warmiński and Teter, 2012). The coefficients in equilibrium equations (2.7) are given in papers: Sridharan et al. (1984), Kołakowski (1996), Schokker (1996), Kołakowski et al.) (1999), Teter and Kołakowski (2004), Teter (2007, 2011), Kołakowski and Kubiak (2005), Kołakowski and Królak (2006), Kubiak (2007, 2013), Warmiński and Teter (2012), Kołakowski and Mania (2013). In the former parts of this paper, in relationships $(2.7), \lambda_{r}$ has been replaced with $\sigma_{r}$, whereas $\lambda$ with $\sigma$, correspondingly.

The initial conditions have been assumed in the form

$$
\zeta_{r}(t=0)=0 \quad \zeta_{r, t}(t=0)=0
$$

In equation (2.7), the quantity $\Omega_{r}^{2}$ depending on the values of $\sigma / \sigma_{r}$ can take the following values:

- if $\sigma / \sigma_{r}<1$, then $\Omega_{r}^{2}=\omega_{r}^{2}\left(1-\sigma / \sigma_{r}\right)>0$, where $\Omega_{r}$ can be called the equivalent angular velocity. The linear general solution to equation (2.9) in the case of an ideal structure (that is to say, for $\left.\zeta_{r}^{*}=0\right)$ are trigonometric functions;

- if $\sigma / \sigma_{r}=1$, then $\Omega_{r}^{2}=0$;

- if $\sigma / \sigma_{r}<1$, then $\Omega_{r}^{2}<0$, where $\Omega_{r}$ can be called the equivalent growing function and then the linear general solution to equation (2.7) for $\zeta_{r}^{*}=0$ are hyperbolic functions.

The nonlinear static stability (i.e., for $\zeta_{r, t t}$ in (2.7)) of thin-walled multilayer structures in the first order approximation of Koiter's theory is solved with the modified analytical-numerical method (ANM) presented by Kołakowski and Królak (2006). The analytical-numerical method (ANM) should consider also the second order approximation of the theory in the analysis of postbuckling of elastic composite structures. The second order postbuckling coefficients were estimated with the semi-analytical method (SAM) (Kołakowski, 1996) modified by the solution method in Kołakowski and Mania (2013). In the semi-analytical method (SAM) for static problems, one postulates to determine approximated values of the coefficients $b_{\text {rrrr }}$ in (2.7) on the 
basis of the linear buckling problem. This approach allows the values of the coefficients $a_{p q r}$ in (2.7) - according to the applied nonlinear Byskov and Hutchinson theory (Byskov and Hutchinson, 1977) - to be determined precisely. The natural frequencies have been determined analogously as in Teter and Kołakowski (2003), whereas the problem of interactive dynamic buckling (2.7) has been solved by means of the Runge-Kutta numerical method modified by Hairer and Wanner.

In the present paper, we assume that the buckling modes are the same as the vibration modes, so the solution to the eigenvalue problem is sought for various values of the $m$-th harmonic. Values of the natural frequencies are determined taking into account all components of inertia forces.

For static problems, Koiter and van der Neut (Kubiak, 2013) have proposed a technique in which an interaction of the overall mode with two local modes having the same wavelength (i.e., a three-mode approach $J=3$ ) has been considered. The fundamental local mode is henceforth called "primary" and the nontrivial higher mode (having the same wavelength as the "primary" one), corresponding to the mode triggered by the overall long-wave mode, is called "secondary". In total energy, the coefficients of the cubic terms $\zeta_{1} \zeta_{2}^{2}, \zeta_{1} \zeta_{3}^{2}$ and $\zeta_{1} \zeta_{2} \zeta_{3}$ (where $\zeta_{j}$ is the amplitude of the $r$-th buckling mode and the index is: 1 for the global mode, 2 for the primary local buckling mode, and 3 for the secondary local mode) are the key terms governing the interaction. In the analysis of the column with doubly symmetric cross-sections, the coefficients of $\zeta_{1} \zeta_{2}^{2}$ and $\zeta_{1} \zeta_{3}^{2}$ terms - the coefficients $a_{p q r}$ of non-linear system of equations (2.7) - vanish.

In the paper by Kołakowski et al. (2015), nonlinear Koiter's theory has been used to explain the effect of the imperfection sign (sense) on local postbuckling equilibrium paths of plates made of functionally graded materials (FGMs). In the case of the FG plate, nonzero first-order sectional inner forces that cause an occurrence of nonzero postbuckling coefficients responsible for sensitivity of the system to imperfections appear. It results in the fact that postbuckling equilibrium paths of plate structures made of FGMs are unsymmetrically stable. This explains differences in the plate response dependence on the imperfection sign (sense). On the other hand, in Kołakowski and Mania (2015), an analysis of the influence of the imperfection sign on the dynamic postbuckling equilibrium paths of the FG square plates has been continued.

In the numerical calculations of dynamic interactive buckling, a rectangular shape of the in-plane pulse loading (i.e., $\sigma(t)=\sigma_{D}$ for $0 \leqslant t \leqslant T_{1}$ and $\sigma(t)=0$ for $T<t_{1}$ ) equal to the fundamental period of the natural frequency $T_{1}=2 \pi / \omega_{1}$ is considered.

The prebuckling solution to the FG plate consisting of homogenous fields is assumed as in Kołakowski and Królak (2006)

$$
u^{(0)}=\left(\frac{l}{2}-x\right) \Delta \quad v^{(0)}=y \Delta \frac{A_{12}}{A_{22}} \quad w^{(0)}=0
$$

where $\Delta$ is the actual loading. This loading of the zero state is specified as a product of the unit loading and the scalar load factor $\Delta$.

Taking into account relationship (2.3), the inner sectional forces of the prebuckling (i.e., unbending) state for the assumed homogeneous field of displacements (2.9) are expressed by the following relationships before the redistribution of forces in the plate due to plate deformations (Kołakowski et al., 2015; Kołakowski and Mania, 2015; Kołakowski, 2016)

$$
\begin{array}{ll}
N_{x}^{(0)}=-\left(A_{11}-\frac{A_{12}^{2}}{A_{22}}\right) \Delta & M_{x}^{(0)}=-\left(B_{11}-B_{12} \frac{A_{12}}{A_{22}}\right) \Delta \\
N_{y}^{(0)}=0 & M_{y}^{(0)}=-\left(B_{12}-B_{22} \frac{A_{12}}{A_{22}}\right) \Delta \\
N_{x y}^{(0)}=0 & M_{x y}^{(0)}=0
\end{array}
$$


The assumed field of displacements and the field of inner forces, corresponding to it for the prebuckling state, fulfil the equilibrium equations for the zero state as an identity.

In Kołakowski and Królak (2006), an unbending, prebuckling state, i.e., a distribution field of the zero state according to (2.9), has been assumed. Dependence (2.3) for the zero state (i.e., prebuckling) takes the form

$$
\overline{\mathbf{N}}_{0}=\left\{\begin{array}{l}
N^{(0)} \\
M^{(0)}
\end{array}\right\}=\left[\begin{array}{ll}
\mathbf{A} & \mathbf{B} \\
\mathbf{B} & \mathbf{D}
\end{array}\right]\left\{\begin{array}{c}
\boldsymbol{\varepsilon}^{(0)} \\
\mathbf{0}
\end{array}\right\}
$$

It results in an occurrence of the nonzero inner sectional forces $(2.10) N_{x}^{(0)}, M_{x}^{(0)}, M_{y}^{(0)}$ in the FG plate for the zero state. Special attention should be paid to the fact that nonzero magnitudes of the sectional moments $M_{x}^{(0)}$ and $M_{y}^{(0)}$ appear due to the effects of deformations of the middle surface (i.e., membrane deformations) resulting from the nontrivial coupling submatrix $\mathbf{B}$ and deformations of the middle surface, and not due to an appearance of curvatures of the middle surface. These moments affect obviously the values of critical loads and the values of postbuckling coefficients (Kołakowski et al., 2015; Kołakowski and Mania, 2015).

\section{Analysis of the results}

Prismatic thin-walled beam-columns with quadratic cross-sections subjected to an axial pulse compression have been considered. The columns are made of the same FGM subject to Hooke's law. A schematic view of the column and its dimensions are shown in Fig. 1.
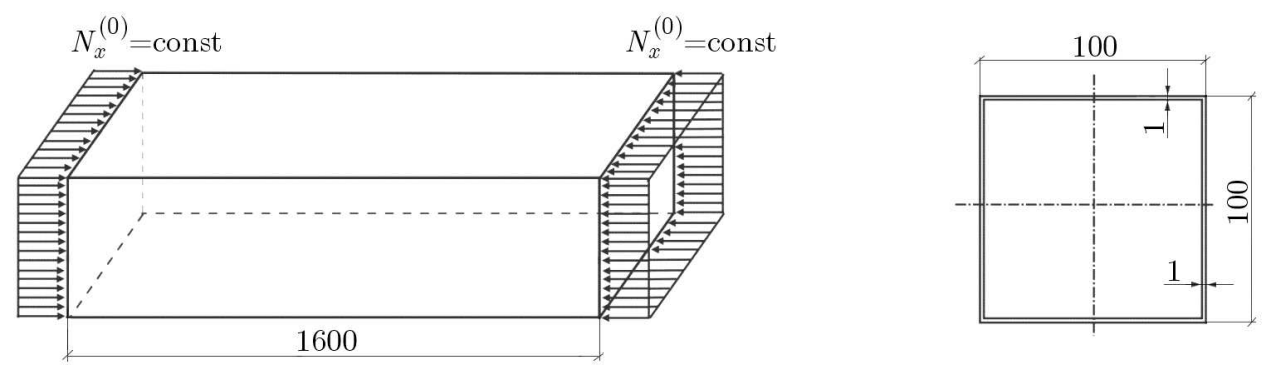

Fig. 1. Thin-walled cross-section of the beam-column

Identically as in Kołakowski et al. (2015), Kołakowski and Mania (2015) two different materials the columns are made of have been considered, namely:

- isotropic material - steel - with the following material properties: Young's modulus $E=210 \mathrm{GPa}$, Poisson's ratio $\nu=0.3$, and density $\rho=7850 \mathrm{~kg} / \mathrm{m}^{3}$;

- Al-TiC functionally graded material (FGM). The component material properties are given: Al Young's modulus: $E_{m}=69 \mathrm{GPa}$, TiC Young's modulus: $E_{c}=480 \mathrm{GPa}$, Al Poisson's ratio: $\nu_{m}=0.33$, TiC Poisson's ratio: $\nu_{c}=0.20$, Al density: $\rho_{m}=2700 \mathrm{~kg} / \mathrm{m}^{3}, \mathrm{TiC}$ density: $\rho_{c}=4920 \mathrm{~kg} / \mathrm{m}^{3}$. The fraction exponent $q(2.2)$ is equal to 1.0 .

Three variants of the beam-column structure have been considered, namely:

- variant I - isotropic column (the so-called reference variant);

- variant II - QCM FG column (ceramics inside the cross-section, metal outside);

- variant III - QMC FG column (metal inside the cross-section, ceramics - outside).

The isotropic beam-column (variant I) has symmetrical stable postbuckling local equilibrium paths, whereas the FG column (variants II and III) has nonsymmetrical stable postbuckling local 
equilibrium paths (Kołakowski et al., 2015; Kołakowski and Mania, 2015). Due to the above aspects, two variants of the FG beam-column - QCM and QMC - have been considered.

For each of these three structural variants, values of critical stresses $\sigma_{r}$ : the global (Euler) buckling mode $\sigma_{1}$, the lowest primary local buckling mode $\sigma_{2}$ and the secondary local buckling mode $\sigma_{3}$ have been determined, respectively. Moreover, numbers of halfwaves $m$ corresponding to the critical loads have been given. The global mode occurs at $m=1$ and the local modes at $m>1$. Also, values of frequencies of free vibrations $\omega_{r}$ and a period of free vibrations $T_{r}$ corresponding to the buckling modes under analysis have been defined as well.

An interactive dynamic response of beam-columns to the load whose duration corresponds to the fundamental period of flexural free vibrations of unloaded columns $T_{1}=2 \pi / \omega_{1}$ has been investigated.

The assumed time of the rectangular load pulse duration corresponds to the beam-column quasi-static load for local modes (i.e., $T_{2}>2 T_{1}, T_{3}>2 T_{1}$ ). The tracing time of the structure dynamic response assumed as $t^{*}=1.5 T_{1}$ has been analysed. The dynamic load factor $D L F$ is defined as a ratio of the dynamic load to the minimal critical value of the static load, $D L F=\sigma_{D} / \min \left(\sigma_{1}, \sigma_{2}, \sigma_{3}\right)=\sigma_{D} / \sigma_{2}$.

In Budiansky and Hutchinson (1966), it has been shown for the two-mode approach that when the frequencies of free vibrations differ at least twice, then in this case the dynamic term corresponding to a higher frequency of free vibrations can be neglected in the equations of motion. On the other hand, in Kołakowski (2016), attention has been paid to unexpected aspects of interactive dynamic buckling in the case of beam-columns having one axis of symmetry of the cross-section. Here, in order to show qualitatively different dynamic responses, a two- or three-mode approach could be applied. It has allowed the uncoupling of the equations of motion for the case of the inner combined resonance, when $\left(\omega_{1}+\omega_{2}\right) / \omega_{3} \approx 1$ (Nayfeh and Mook, 1979).

It is not possible to use this approach in the present study because the interaction occurs only via the coefficient of the cubic term $\zeta_{1} \zeta_{2} \zeta_{3}$ in total energy (2.6) governing the mode interaction. Therefore, an approach described in Budiansky and Hutchinson (1966) has been applied. The secondary local buckling mode in the case of static issues is a supplementary mode that enables one to account for the effect of the primary local mode on the global flexural buckling mode.

In the further part of the study, it has been assumed that when "complete" three dynamic equations of motion (2.7) are considered, then the case is referred to as case I. When the dynamic term is neglected for the primary local buckling mode (i.e., for $r=2$ ), that is to say, when $\zeta_{2, t t}=0$ is assumed in equations (2.7) - it is case II, whereas when the term $\zeta_{3, t t}=0$ is neglected for the secondary local mode (i.e., for $r=3$ ) - case III.

When the dynamic term is neglected in (2.7), which corresponds to case II, one of the equations is a static equation of the third order with respect to the amplitude of deflection $\zeta_{2}$, which is solved on the basis of analytical formulae. Due to this, wrong conditions in the equations could be avoided and the numerical solution could be stabilised. It means that in the formula for total energy of the system (2.6), the expression for kinetic energy $0.5 m_{2} \zeta_{2, t}^{2}$ corresponding to the primary local mode (case II) could be neglected. Analogously, in case III - the expression for the energy $0.5 m_{3} \zeta_{3, t}^{2}$ corresponding to the secondary local mode (i.e., $\zeta_{3}$ ) could be omitted.

Further on in the study, the following index notations of dimensionless amplitudes of deflection have been introduced for the cases under consideration, namely: case $\mathrm{I}-\zeta_{r}$ for $r=1,2,3$; case II $-\widehat{\zeta}_{r}$ for $r=1,2,3$; case III $-\widetilde{\zeta}_{r}$ for $r=1,2,3$. In the calculations of dynamic stability, the following values of imperfections have been assumed: $\zeta_{1}^{*}=1.0, \zeta_{2}^{*}=0.2, \zeta_{3}^{*}=0.1$. Contrary to other works by the authors (e.g., Kołakowski, 1996, 2016; Kołakowski et al., 1999; Teter and Kołakowski, 2001, 2004, 2005; Teter, 2001, 2007, 2011; Kołakowski and Kubiak, 2005; Kołakowski and Królak, 2006; Kubiak, 2007; Kołakowski and Mania, 2013; Kubiak, 2013), the most unfavourable combination of initial imperfection signs $\zeta_{r}^{*}$ (for $r=1,2,3$ ) has not been assumed 
to make the effects of nonsymmetrical stable postbuckling equilibrium path and the influence of the connection between the adjacent plates of the cross-section more visible.

Values of the critical dynamic load factors $D L F_{c r}$ have been determined from the Budiansky-Hutchinson criterion (Budiansky amd Hutchinson, 1977; Kubiak 2007, 2013) in which it is assumed that the loss of dynamic stability occurs when the velocity with which the displacements grow is the highest for a certain force amplitude. The values of $D L F_{c r}$ presented correspond with some accuracy to the maximum values of deflections $\max \left(\zeta_{1}\right)$ within the applicability of the assumed theory (i.e., the total maximum deflection of the column is at least a hundred times as high as the cross-section wall thickness), and not to asymptotic values (Kubiak, 2013).

\subsection{Variant $\mathbf{I}-$ isotropic columns}

Variant I has been assumed as the reference one because in the case of an isotropic column, the plates constituting the cross-section are characterized by the symmetry of cross-sectional uniformity (i.e., coupling stiffness matrix $\mathbf{B}=\mathbf{0}$ in (2.3)).

Table 1. Solutions to the eigenproblem for an isotropic column - variant I (reference)

\begin{tabular}{|c|c|c|c|c|}
\hline$r$ & $\sigma_{r}[\mathrm{MPa}]$ & $m[-]$ & $\omega_{r}[\mathrm{rad} / \mathrm{s}]$ & $T_{r}[\mathrm{~ms}]$ \\
\hline \hline 1 & 1185.6 & 1 & 761 & 8.256 \\
\hline 2 & 72.28 & 16 & 3013 & 2.085 \\
\hline 3 & 103.66 & 16 & 3609 & 1.741 \\
\hline
\end{tabular}

First, the eigenvalues of the problem given in Table 1 have been determined, namely: values of critical stresses corresponding to the global Euler buckling $\sigma_{1}$, the lowest primary local buckling mode $\sigma_{2}$ and the secondary local buckling mode $\sigma_{3}$, the frequencies of free vibrations and the periods corresponding to them, as well as a number of halfwaves $m$ of the eigenmodes along the longitudinal direction. The ratios of frequencies of free vibrations are equal to: $\omega_{2} / \omega_{1}=3.96$, $\omega_{3} / \omega_{1}=4.74, \omega_{3} / \omega_{2}=1.197,\left(\omega_{1}+\omega_{2}\right) / \omega_{3}=1.045$, respectively. In Fig. 2a, absolute values of the maximum amplitudes of global defections (for $\mathrm{r}=1$ ) (i.e., $\zeta_{1}, \widehat{\zeta}_{1}, \widetilde{\zeta}_{1}$ ) as a function of $D L F$ for cases I-III under consideration, whereas in Fig. $2 \mathrm{~b}$ - for local deflections of the modes $\zeta_{2}, \widehat{\zeta}_{3}$ and $\widetilde{\zeta}_{2}$ for cases I, II and III are shown, correspondingly.
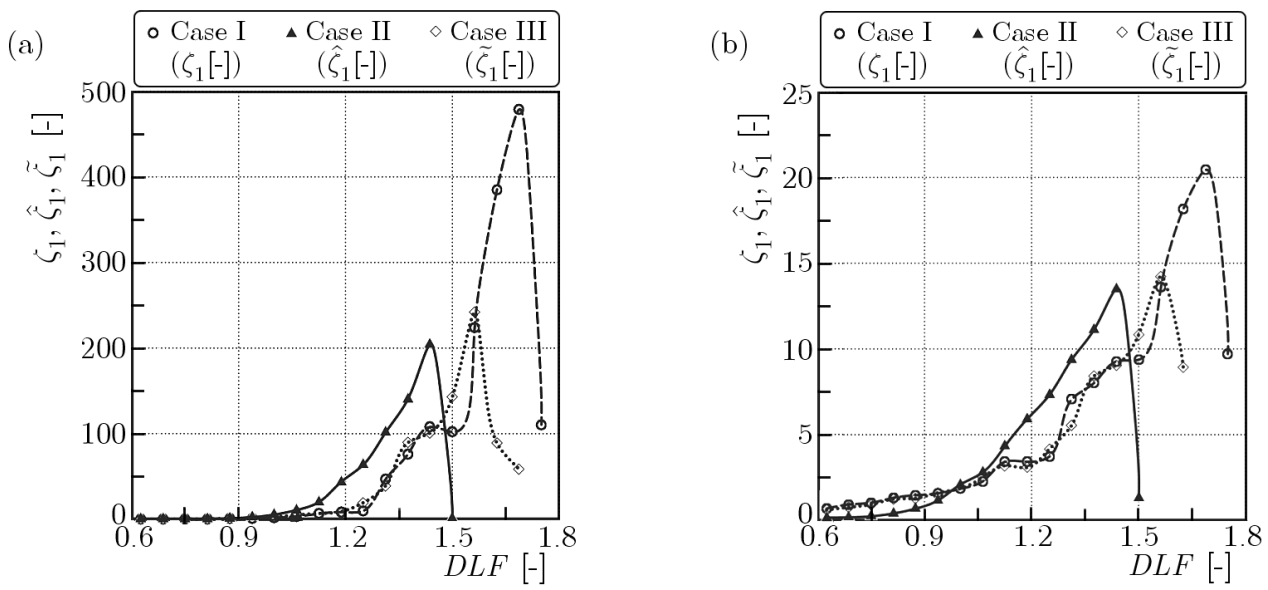

Fig. 2. Maximum dimensionless global deflections as a function of $D L F$ for an isotropic column (variant I): (a) global modes, (b) local modes

Quantative differences in the component amplitudes of global deflections (i.e., $\zeta_{1}, \widehat{\zeta}_{1}$ and $\widetilde{\zeta}_{1}$ ) and the local ones (i.e., $\zeta_{2}, \widehat{\zeta}_{3}$ and $\widetilde{\zeta}_{2}$ ) can be observed. As can be easily seen in these two figures, the maximum values of the amplitudes of the global and local deflection for each case under 
investigation occur for the same values of $D L F$ (i.e., $D L F \approx 1.69$ for case I, $D L F \approx 1.44$ for case II, and $D L F \approx 1.56$ for case III, respectively).

When $D L F=1.434$, then the amplitude of dynamic load is $\sigma_{D}=\sigma_{3}$ and, moreover, $\sigma_{3} / \sigma_{2}=1.434$ (Table 1 ). When $t \leqslant T_{1}$, then for $1.0 \leqslant D L F<1.434$ and $\Omega_{1}^{2}>0, \Omega_{2}^{2}<0$, $\Omega_{3}^{2}>0$, whereas for $D L F>1.434, \Omega_{1}^{2}>0, \Omega_{2}^{2}<0, \Omega_{3}^{2}<0$, respectively.

The maximum value $\max \left(\zeta_{1}\right)$ is more than twice as high as $\max \left(\widehat{\zeta}_{1}\right)$ and $\max \left(\widetilde{\zeta}_{1}\right)$, whereas the differences between $\max \left(\widehat{\zeta}_{1}\right)$ and $\max \left(\widetilde{\zeta}_{1}\right)$ are equal to approx. $20 \%$. The differences between the maximum values of local amplitudes (i.e., $\max \left(\zeta_{2}\right), \max \left(\widetilde{\zeta}_{2}\right)$ and $\left.\max \left(\widehat{\zeta}_{3}\right)\right)$ are not so significant. The value $\max \left(\zeta_{2}\right)$ is more than $30 \%$ higher than the $\max \left(\widetilde{\zeta}_{2}\right)$ and $\max \left(\widehat{\zeta}_{3}\right)$. The maximum values of global deflections are by one order of magnitude higher than for local deflections.

As can be seen in Fig. 2 for case II, the deflections $\widehat{\zeta}_{1}$ and $\widehat{\zeta}_{3}$ for $D L F>1.7$ are many times lower than the deflections in cases I and III.

For case II, the lowest value of $D L F_{c r}$ and the lowest value $\max \left(\widehat{\zeta}_{1}\right) \approx 200$ for $D L F \approx 1.44$ have been obtained (Fig. $2 \mathrm{a}$ ), which correspond to: $\sigma_{D} \approx \sigma_{3}, \sigma_{3} / \sigma_{2}=1.434$ and $\Omega_{3}^{2} \approx 0$.

When $t \leqslant T_{1}$ and for $D L F=1.56$ in case III, we have $\max \left(\widetilde{\zeta}_{1}\right) \approx 250, \Omega_{1}=723, \Omega_{2}=2259$ and $\Omega_{3}=1081$, and for $D L F=1.69$ in case I, we have $\max \left(\zeta_{1}\right)<500, \Omega_{1}=720, \Omega_{2}=2498$ and $\Omega_{3}=1518$. When $T_{1}<t \leqslant t^{*}$, we have $\left(\omega_{1}+\omega_{2}\right) / \omega_{3}=1.045$.

The critical values of $D L F_{c r}$, according to the Budiansky-Hutchinson criterion assumed in this study, are equal for the individual cases to: case I $-D L F_{c r}=1.44$, case II $-D L F_{c r}=1.31$, case III $-D L F_{c r}=1.44$.

The duration of the rectangular load pulse is equal to $T_{1}=8.256 \mathrm{~ms}$. At the response tracing time $T_{1}<t \leqslant t^{*}$, the system is not subject to load any longer and performs free unsteady vibrations in the transient period. Thus, the values of maximum amplitudes are attained in this time range for all the time functions described.

\subsection{Variant II - QCM functionally graded column}

Two variants of the FG column structure have been assumed. It has followed from two aspects. The first one is such that it is possible to make such columns in two ways. The ceramic surface is resistant to high temperatures. Variant II (i.e., QCM) protects the outer surface of the cross-section against high temperature, whereas variant III (QMC) protects the inner surface. The second aspect results from the fact that FG structures have nonsymmetrical stable postbuckling equilibrium paths (Kołakowski et al., 2015; Kołakowski and Mania, 2015). Hence, the way the component plates of the FG column are connected exerts an influence on the postbuckling behavior of the structure. For FG columns, identical calculations have been conducted as for variant I (the reference one). For variant II (QCM), the results of calculations of the eigenproblem have been presented in Table 2 . The ratios of frequencies of free vibrations are equal to: $\omega_{2} / \omega_{1}=3.57, \omega_{3} / \omega_{1}=4.27, \omega_{3} / \omega_{2}=1.196,\left(\omega_{1}+\omega_{2}\right) / \omega_{3}=1.070$, correspondingly.

Table 2. Solutions to the eigenproblem for a QCM functionally graded column - variant II

\begin{tabular}{|c|c|c|c|c|}
\hline$r$ & $\sigma_{r}[\mathrm{MPa}]$ & $m[-]$ & $\omega_{r}[\mathrm{rad} / \mathrm{s}]$ & $T_{r}[\mathrm{~ms}]$ \\
\hline \hline 1 & 1606.3 & 1 & 1273 & 4.935 \\
\hline 2 & 79.81 & 16 & 4546 & 1.382 \\
\hline 3 & 114.17 & 16 & 5437 & 1.155 \\
\hline
\end{tabular}

Figure 3 show the absolute maximum values of global and local deflections versus $D L F$ for the three cases under consideration, respectively. Similarly as for variant I (i.e., the reference one - isotropic), quantative differences in the values of global (i.e., $\zeta_{1}, \widehat{\zeta}_{1}$ and $\widetilde{\zeta}_{1}$ ) and local (i.e., $\zeta_{2}, \widehat{\zeta}_{3}$ and $\widetilde{\zeta}_{2}$ ) amplitudes can be seen easily. 

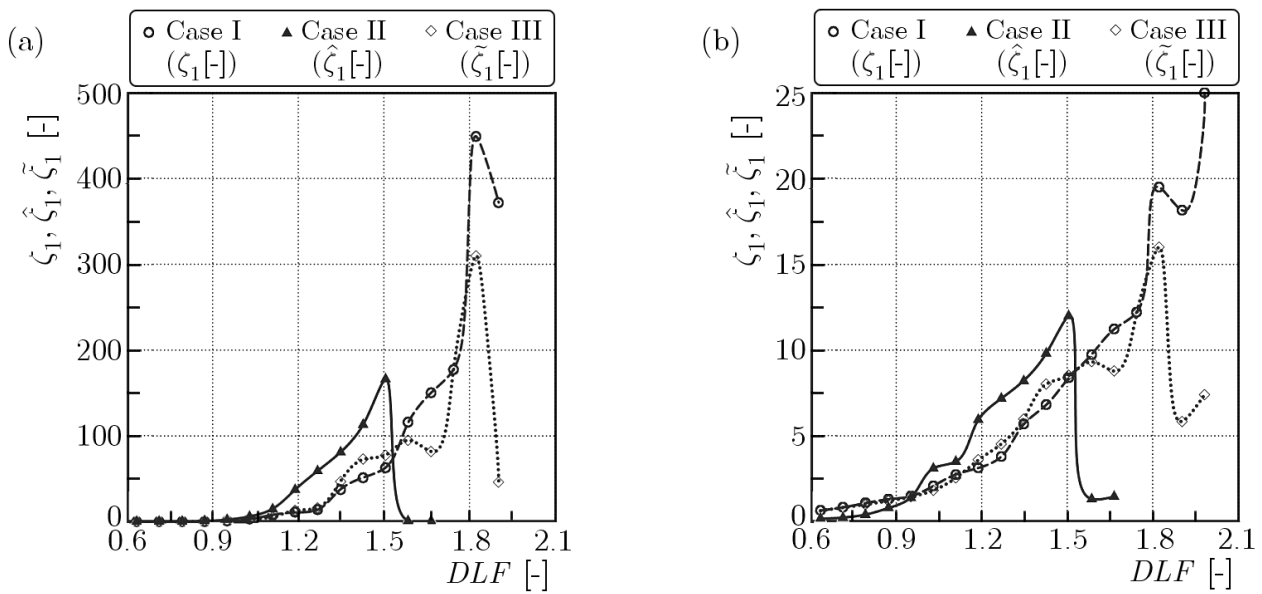

Fig. 3. Maximum dimensionless global deflections as a function of $D L F$ for variant II: (a) global modes, (b) local modes

For each case, the maximum value of global and local deflection occurs for the same values of $D L F$ (i.e., case I $-D L F=1.82$, case II $-D L F=1.51$ and case III $-D L F=1.82$ ). When $D L F=1.4307$, then we have $\sigma_{3} / \sigma_{2}=1.4307$. When $t \leqslant T_{1}$, then for $1.0<D L F<1.4307$ we have $\Omega_{1}^{2}>0, \Omega_{2}^{2}<0$ and $\Omega_{3}^{2}>0$, whereas for $D L F>1.4307$, we have, $\Omega_{1}^{2}>0, \Omega_{2}^{2}<0$ and $\Omega_{3}^{2}<0$, correspondingly.

The maximum values $\max \left(\zeta_{1}\right)$ and $\max \left(\widetilde{\zeta}_{1}\right)$ are twice as high as $\max \left(\widehat{\zeta}_{1}\right)$, and $\max \left(\zeta_{1}\right) / \max \left(\widetilde{\zeta}_{1}\right) \approx 1.5$. The differences between the maximum values of local deflections, i.e., $\zeta_{2}, \widehat{\zeta}_{3}$ and $\widetilde{\zeta}_{2}$, are not so high and they do not exceed 1.5 times. The values $\max \left(\zeta_{1}\right), \max \left(\widehat{\zeta}_{1}\right)$, $\max \left(\widetilde{\zeta}_{1}\right)$ for global modes are higher by one order of magnitude than $\max \left(\zeta_{2}\right), \max \left(\zeta_{3}\right), \max \left(\widehat{\zeta}_{3}\right)$, $\max \left(\widetilde{\zeta}_{2}\right)$ for local modes.

In Fig. 3, for the duration of load (i.e., for $t \leqslant T_{1}$ ), we have $\Omega_{1}=1224, \Omega_{2}=3235$ and $\Omega_{3}=1251$ for $D L F=1.51$, whereas for $D L F=1.81-\Omega_{1}=1213, \Omega_{2}=4125$ and $\Omega_{3}=2849$, respectively. For $T_{1}<t \leqslant t^{*}$, we have $\left(\omega_{1}+\omega_{2}\right) / \omega_{3}=1.070$.

The critical values $D L F_{c r}$ are equal to, respectively: case I $-D L F_{c r}=1.56$, case II $D L F_{c r}=1.40$, case III $-D L F_{c r}=1.69$. For $D L F=1.11, D L F=1.27$ and cases I and III, the maximum values of global deflections $\max \left(\zeta_{1}\right)$ and $\max \left(\widetilde{\zeta}_{1}\right)$ are attained at $T_{1}<t \leqslant t^{*}$, that is to say, after the load pulse duration. The values of local deflections $\max \left(\zeta_{2}\right), \max \left(\zeta_{3}\right)$ and $\max \left(\widehat{\zeta}_{3}\right)$ are approximately three times lower than global deflections. For the two values of $D L F$ under consideration in case II, the global deflections $\widehat{\zeta}_{1}$ grow monotonously in practice and $\max \left(\widehat{\zeta}_{1}\right)$ is twice as high as $\max \left(\zeta_{1}\right)$ and $\max \left(\widetilde{\zeta}_{1}\right)$. When $D L F=1.35$, global deflections increase monotonously during the whole response tracing time for all the three cases.

Similarly as for variant I, the maximum values of global deflations (i.e., $\max \left(\zeta_{1}\right), \max \left(\widehat{\zeta}_{1}\right)$, $\left.\max \left(\widetilde{\zeta}_{1}\right)\right)$ are attained when the load impulse finishes for the presented time functions.

\subsection{Variant III - QMC functionally graded column}

In Table 3, the calculation results of the eigenproblem for variant III (QMC) are presented. The ratios of frequencies of free vibrations are equal to: $\omega_{2} / \omega_{1}=3.53, \omega_{3} / \omega_{1}=4.24, \omega_{3} / \omega_{2}=$ 1.199, $\left(\omega_{1}+\omega_{2}\right) / \omega_{3}=1.069$, respectively. As can be easily seen, the eigenvalues for both variants of the FGM column (compare Tables 3 and 2 ) are very close to each other.

In Fig. 4, the absolute values of maximum amplitudes of global (i.e., $\zeta_{1}, \widehat{\zeta}_{1}$ and $\widetilde{\zeta}_{1}$ ) and local (i.e., $\zeta_{2}, \widehat{\zeta}_{3}$ and $\widetilde{\zeta}_{2}$ ) deflections versus the coefficient $D L F$ for the three cases under consideration are presented. Analogously as for variants I and II, quantative differences for components of global and local amplitudes can be observed. As can be easily seen in Fig. 4, the maximum value 
Table 3. Solutions to the eigenproblem for a QCM functionally graded column - variant III

\begin{tabular}{|c|c|c|c|c|}
\hline$r$ & $\sigma_{r}[\mathrm{MPa}]$ & $m[-]$ & $\omega_{r}[\mathrm{rad} / \mathrm{s}]$ & $T_{r}[\mathrm{~ms}]$ \\
\hline \hline 1 & 1616.6 & 1 & 1277 & 4.920 \\
\hline 2 & 78.77 & 16 & 4516 & 1.391 \\
\hline 3 & 113.27 & 16 & 5415 & 1.160 \\
\hline
\end{tabular}

of global and local deflection occurs for the same values of $D L F$ in cases II and III (case II $D L F=1.45$, case III $-D L F=1.85)$. In case I, the amplitudes of deflections grow monotonously for $D L F<2.0$. For $D L F=2.0$, we have $\max \left(\zeta_{1}\right)>500$ and $\max \left(\zeta_{2}\right)>25$. For $D L F=1.4383$, we have $\sigma_{3} / \sigma_{2}=1.4383$. When $t \leqslant T_{1}$, then for $1.0<D L F<1.4383$, we have $\Omega_{1}^{2}>0$, $\Omega_{2}^{2}<0$ and $\Omega_{3}^{2}>0$, whereas at $D L F>1.4383, \Omega_{1}^{2}>0, \Omega_{2}^{2}<0$ and $\Omega_{3}^{2}<0$, respectively. The lowest values of maximum deflections are for case II, that is to say, for $\max \left(\widetilde{\zeta}_{1}\right)$ and $\max \left(\widehat{\zeta}_{3}\right)$, analogously as for variant II.
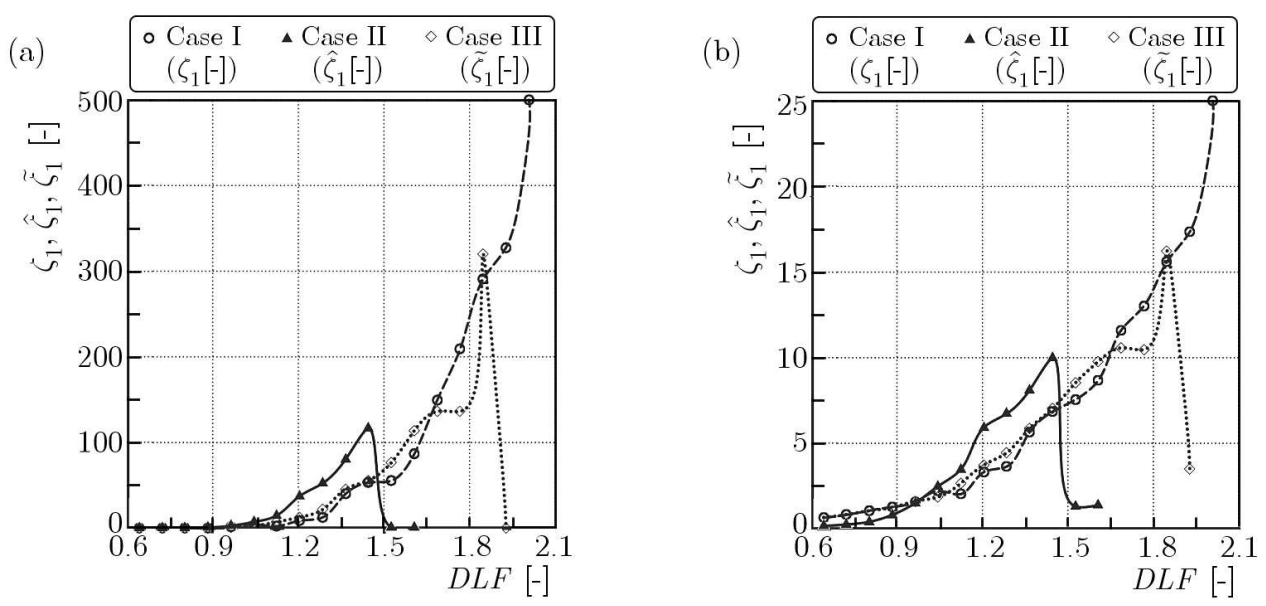

Fig. 4. Maximum dimensionless global deflections as a function of $D L F$ for variant III: (a) global modes, (b) local modes

In Fig. 4, under the dynamic loading (for $t \leqslant T_{1}$ ) for $D L F=1.45$, we have $\Omega_{1}=1231$, $\Omega_{2}=3015$ and $\Omega_{3} \approx 0$, whereas for $D L F=1.85-\Omega_{1}=1218, \Omega_{2}=4157$ and $\Omega_{3}=28488$, respectively. At $T_{1}<t \leqslant t^{*}$, we have $\left(\omega_{1}+\omega_{2}\right) / \omega_{3}=1.069$. The critical values of $D L F_{c r}$ are equal to: case I $-D L F_{c r}=1.56$, case II $-D L F_{c r}=1.69$, case III $-D L F_{c r}=1.40$. When $t \leqslant T_{1}$, then for the assumed values of $D L F$, we have $\Omega_{1}^{2}>0, \Omega_{2}^{2}<0$ and $\Omega_{3}^{2}>0$. For the three values of $D L F$ under consideration and, simultaneously, for the three cases, the maximum global deflection during the response tracing time (i.e., $\left.0 \leqslant t \leqslant t^{*}\right)$ occurs for case II $-\max \left(\widehat{\zeta}_{1}\right)$. When the time functions for variants II and III are compared, some insignificant differences can be observed. The maximum values of global deflections (i.e., $\left.\max \left(\zeta_{1}\right), \max \left(\widehat{\zeta}_{1}\right), \max \left(\widetilde{\zeta}_{1}\right)\right)$ take place for $T_{1}<t \leqslant t^{*}$.

\subsection{Comparison of all variants under analysis}

For all variants under consideration (i.e., I-III), diagrams of amplitudes of global deflections are similar for cases I and III, whereas significant differences can be observed for case II. It corresponds to the case when the dynamic term $\zeta_{2, t t}=0$ is neglected in $(2.7)$, which has a visible effect on the equations of motion. Moreover, for $D L F>1.45$, we have $\Omega_{1}^{2}>0, \Omega_{2}^{2}<0$ and $\Omega_{3}^{2}<0$. It means that the amplitudes of local deflections grow exponentially and become dominating in a short time. A dynamic interaction of the global mode and the local ones results in a very dramatic increase in the amplitudes of deflections. 
For the time functions under consideration for all variants and cases and the assumed values of load coefficients $D L F$, the amplitudes of global deflections attain their maximum values after the pulse load finishes (e.g., for $T_{1}<t \leqslant t^{*}$ ). For this time range, free unsteady vibrations occur in the transient process. To the authors' knowledge, the only phenomenon that can explain this effect is the inner combined resonance which takes place accordingly to the theory of vibration (Nayfeh and Mook, 1979) for steady processes, correspondingly, in the cases when $\left(\omega_{1}+\omega_{2}\right) / \omega_{3} \approx 1.0$. The three cases under analysis comply with the above-mentioned condition. A change in the tracing time of the dynamic response $t^{*}$ can exert an influence on the maximum values of amplitudes of deflections. In the study, a long enough tracing time has been assumed, as it is equal to $t^{*}=1.5 T_{1}$. However, shortening of this time (e.g., up to $t^{*}=1.3 T_{1}$ ) will not exert any influence on the general conclusions drawn here.

\section{Conclusions}

Dynamic interactive buckling of thin-walled FGM columns with a square cross-section is discussed. Three cases are considered. Case I corresponds to analysis of the dynamic response of the FG structure for "complete" equations of motion (2.7), case II refers to the situation when the dynamic effect is neglected for the primary local buckling mode (i.e., $r=2$ ), whereas case III for the secondary local mode (i.e., $r=3$ ). Such an approach has been pointed out by Budiansky and Hutchinson (1966). When the structure has two axes of symmetry of the cross-section, the interactive buckling occurs only via the coefficient of the cubic term $\zeta_{1} \zeta_{2} \zeta_{3}$ in total potential energy (2.6). The solution method applied in this study has not been so efficient as in the case of the trapezoidal cross-section with one axis of symmetry. In Kołakowski (2016), however, a quite different approach to the solution of the problem has been assumed. Thus, the above conclusions should be subject to further, thorough investigations.

\section{Acknowledgements}

This publication is a result of the research works carried out within the project subsidized over the years 2012-2015 by the state funds designated for the National Science Centre (NCN UMO-2011/01/B/ST8/07441).

\section{References}

1. Ari-Gur J., Simonetta S.R., 1979, Dynamic pulse buckling of rectangular composite plates, Composites Part B, 28B, 301-308

2. Birman V., BYRD L.W., 2007, Modeling and analysis of functionally materials and structures, Applied Mechanics Review, 60, 195-216

3. Budiansky B., Hutchinson J.W., 1966, Dynamic buckling of imperfection-sensitive structures, Proceedings of the 11th International Congress of Applied Mechanics, Goetler H. (Ed.), Munich, 636-651

4. Byskov E., Hutchinson J.W., 1977, Mode interaction in axially stiffened cylindrical shells, AIAA Journal, 15, 7, 941-948

5. Hui-Shen S., 2009, Functionally Graded Materials - Nonlinear Analysis of Plates and Shells, CRC Press, Taylor \& Francis, London

6. Jones R.M., 1999, Mechanics of Composite Materials, 2nd ed. Taylor \& Francis, London

7. KoŁAKowski Z., 1996, A semi-analytical method for the analysis of the interactive buckling of thin-walled elastic structures in the second order approximation, International Journal of Solids Structures, 33, 25, 3779-3790 
8. Koєakowski Z., 2016, Some aspects of interactive dynamic stability of thin-walled trapezoidal FGM beam-columns under axial load, Thin-Walled Structures, 98, 431-442

9. KoŁakowski Z., Królak M., 2006, Modal coupled instabilities of thin-walled composite plate and shell structures, Composite Structures, 76, 303-313

10. KoŁakowski Z., Królak M., Kowal-Michalska K., 1999, Modal interactive buckling of thin-walled composite beam-columns regarding distortional deformations, International Journal of Engineering Science, 37, 1577-1596

11. KoŁakowski Z., Kubiak T., 2005, Load-carrying capacity of thin-walled composite structures, Composite Structures, 67, 417-426

12. KoŁakowski Z., Mania J.R., 2013, Semi-analytical method versus the FEM for analysis of the local post-buckling, Composite Structures, 97, 99-106

13. KoŁakowski Z., Mania J.R., 2015, Dynamic response of thin FG plates with a static unsymmetrical stable postbuckling path, Thin-Walled Structures, 86, 10-17

14. KoŁakowski Z., Mania R.J., Grudziecki J., 2015, Local nonsymmetric postbuckling equilibrium path in thin FGM plate, Eksploatacja i Niezawodność (Maintenance and Reliability), 17, $135-142$

15. Kubiak T., 2007, Criteria of dynamic buckling estimation of thin-walled structures, Thin-Walled Structures, 45, 10/11, 888-892

16. Kubiak T., 2013, Static and Dynamic Buckling of Thin-Walled Plate Structures, Springer

17. NA K.S., KIM J.H., 2009, Volume fraction optimization of functionally graded composite panels for stress reduction and critical temperature, Finite Elements in Analysis and Design, 45, 845-851

18. NAYFeh A.H., Mook D.T., 1979, Nonlinear Oscilations, Wiley, New York

19. PAnda S., RAY M.C., 2008, Nonlinear finite element analysis of functionally graded plates integrated with patches of piezoelectric fiber reinforced composite, Finite Elements in Analysis and Design, 44, 493-504

20. Petry D., Fahlbusch G., 2000, Dynamic buckling of thin isotropic plates subjected to in-plane impact, Thin-Walled Structures, 38, 267-283

21. ReDdy J.N., 2000, Analysis of functionally graded plates, International Journal for Numerical Methods in Engineering, 47, 663-684

22. Reddy J.N., 2004, Mechanics of Laminated Composite Plates and Shells, CRC Press, London

23. Samsam Shariata B.A., Javaherib R.B.A., Eslami B.A., 2005, Buckling of imperfect functionally graded plates under in-plane compressive loading, Thin-Walled Structures, 43, 1020-1036

24. Schokker A., Sridharan S., Kasagi A., 1996, Dynamic buckling of composite shells, Computers and Structures, 59, 1, 43-55

25. Sridharan S., Benito R., 1984, Columns: static and dynamic interactive buckling, Journal of Engineering Mechanics ASCE, 110, 1, 49-65

26. Teter A., 2007, Static and dynamic interactive buckling of isotropic thin-walled closed columns with variable thickness, Thin-Walled Structures, 45, 10/11, 936-940

27. Teter A., 2011, Dynamic critical load based on different stability criteria for coupled buckling of columns with stiffened open cross-sections, Thin-Walled Structures, 49, 589-595

28. Teter A., KoŁakowski Z., 2001, Lower bound estimation of load-carrying capacity of thin-walled structures with intermediate stiffeners, Thin-Walled Structures, 39, 8, 649-669

29. Teter A., KoŁakowski Z., 2003, Natural frequencies of thin-walled structures with central intermediate stiffeners or/and variable thickness, Thin-Walled Structures, 41, 291-316

30. Teter A., KoŁakowski Z., 2004, Interactive buckling and load carrying capacity of thin-walled beam-columns with intermediate stiffeners, Thin-Walled Structures, 42, 211-254 
31. Teter A., KoŁakowski Z., 2005, Buckling of thin-walled composite structures with intermediate stiffeners, Composite Structures, 69, 4, 421-428

32. VAn Der HeiJden A.M.A. (ED.), 2009, WT Koiter's Elastic Stability of Solids and Structures, Cambridge University Press

33. WArmiński J., Teter A., 2012, Non-linear parametric vibrations of a composite column under uniform compression, Proceedings of the Institution of Mechanical Engineers, Part C: Journal of Mechanical Engineering Science, 226, 8, 1921-1938

Manuscript received Jule 29, 2015; accepted for print September 2, 2015 